\title{
Single embryo transfer and multifetal pregnancy reduction: perception and attitude of women seeking assisted reproduction in Nigeria
}

\author{
Aziken M. E.*, Osaikhuwuomwan J. A., Iribhogbe O. I. \\ Department of Obstetrics and Gynecology, College of Medical Sciences, University of Benin, Benin- City, Nigeria \\ Received: 25 February 2019 \\ Accepted: 02 April 2019 \\ *Correspondence: \\ Dr. Michael E. Aziken, \\ E-mail: michael.aziken@uniben.edu \\ Copyright: () the author(s), publisher and licensee Medip Academy. This is an open-access article distributed under \\ the terms of the Creative Commons Attribution Non-Commercial License, which permits unrestricted non-commercial \\ use, distribution, and reproduction in any medium, provided the original work is properly cited.
}

\begin{abstract}
Background: In-vitro fertilization (IVF) is associated with increased multiple pregnancy and its attendant complications. This study evaluates the attitude and acceptance of single embryo transfer (SET) and multifetal pregnancy reduction (MFPR) by clients assessing assisted reproduction in this region.

Methods: A cross sectional survey of patients selected for IVF was conducted. Information on demography, knowledge of IVF procedure and their perception, attitude and acceptability of multiple pregnancy as well as their knowledge, attitude and perception to single embryo transfer and multifetal pregnancy reduction were extracted for statistical analysis.

Results: Seventy-three women participated in the study. The mean age was 39 years and mean duration of infertility was 8.6 years. Only $3(4.1 \%)$ respondents agreed to have SET as the overwhelming majority (70) 95.9\% preferred 2 or more and they felt the more number of embryo transferred the better the chances of achieving pregnancy. Similarly most respondents, 38.4\% (28) did not accept MFPR. Most respondents considered age (63\%) and duration of infertility $(78.1 \%)$ as major influencing factor for rejecting SET. Over 75\% of respondents said they will still accept multiple embryos transferred despite knowledge of the possible complications.

Conclusions: While most infertile women in our sub region appear to recognize the risks with multiple pregnancy, they are less interested in SET or MFPR because they perceive more embryos transferred as a means to maximize treatment outcome. Government funding, client education and a blastocyst transfer protocol may improve acceptability as well as overall preference for less number of embryos transferred in our environment.
\end{abstract}

Keywords: Embryo transfer, Infertility, In-vitro fertilization, Multifetal pregnancy reduction, Multiple pregnancy, Single embryo transfer

\section{INTRODUCTION}

The art and science of assisted reproduction technique has revolutionized infertility treatment worldwide, such that hitherto, some hopeless infertility conditions are now amenable to rewarding treatment with reported pregnancy rates of $10-18 \%$ and $30-35 \%$ for intrauterine insemination (IUI) and In-vitro Fertilization and Embryo Transfer (IVF-ET) respectively. ${ }^{1-3}$ Consequent on the high cost of treatment (particularly IVF-ET), especially in the resource constraint setting of sub-Saharan Africa, everyone including the provider and the patient is under immense pressure to achieve a high success rate. This quest has encouraged the transfer of multiple embryos at IVF-ET in order to enhance a successful outcome. ${ }^{4-6}$

While transfer of multiple embryos may be associated with increased pregnancy rates per cycle of treatment, it is also a cause of increased rates of multiple pregnancy which is considered as a treatment complication because of its attendant complications and ultimately decrease in the live birth rate. ${ }^{7,8}$ 
Multiple pregnancy, particularly the higher order multiple gestation is associated with increased risk of miscarriage and preterm delivery before 34 weeks of gestation. ${ }^{7-9}$ Prematurity which is the main cause of neonatal morbidity and mortality occurs in almost half of multiple pregnancies. In one of the report, over $40 \%$ of women with twin pregnancies delivered before 37 weeks compared to less than $10 \%$ amongst singletons. The risk of perinatal mortality increased six folds in twins and 1020 folds in triplets compared to singletons. ${ }^{9}$ Even amongst neonatal survivors of preterm delivery, long term complications such as cerebral palsy and neurobehavioral disorders are major concerns to the family and society. ${ }^{10}$

As a result of the increased morbidity and mortality associated with multiple pregnancy, several documents have advocated the transfer of fewer embryos preferably one embryo (single embryo transfer: SET) in selected patients. ${ }^{11,12}$ In 2003, De Sutter et al reported a significant reduction of multiple pregnancy with elective SET without a decline in the pregnancy rate. ${ }^{12}$ There are other reports on the beneficial effects of elective SET in reducing multiple pregnancy rates in IVF programs without dropping the pregnancy rates. ${ }^{8,12,13}$ Titinen and co-workers also reported reduced multiple pregnancy and a better cumulative pregnancy rates when SET is backed by embryo freezing program. ${ }^{14}$ Despite the aforementioned risk of multiple pregnancies and the benefit of transferring fewer embryos, many infertile patients accessing IVF treatment would still desire multiple embryo transfer because of their strong desire to succeed at first attempt as well as the high cost of treatment particularly when there is no government funding. This is particularly true for sub-saharan Africa where in addition there exist a wide spread cultural acceptability and desire for multiple pregnancy hence the tendency for clinicians to encourage multiple embryo transfer at IVF-ET. ${ }^{6,15,16}$

Multifetal pregnancy reduction is another modality to mitigate the prevalence and attendant complications of multiple pregnancy associated with assisted reproduction. Owing to poor uptake of SET by IVF patients, clinicians have advocated multifetatal pregnancy reduction (MFPR) as a final strategy to reduce the morbidity and mortality associated with IVF created multiple pregnancy. ${ }^{17,18}$ MFPR refers to willful termination of further development in one or more fetuses to reduce the number of viable fetuses to two or occasionally one. Despite the reported benefit of MFPR, acceptance of the procedure is still low for several reasons chief amongst these are psychological, sociological and ethical consideration. ${ }^{19}$

IVF services are becoming widespread in Nigeria, with the majority in private facilities and only few in Government institutions albeit still patient funded. The pressure to succeed at first attempt is high amongst clinician and patients' worldwide; particularly in settings where the cost of treatment is solely borne by the patients like is the situation in Nigeria. The need to succeed as soon as possible would therefore impact on how an IVF patient perceives multiple gestation and all the interventions to reduce the complications. As IVF-ET is gaining prominence in Nigeria, it becomes appropriate to evaluate the attitude and acceptance of policies such as single embryo transfer and multifetal pregnancy reduction by clients assessing assisted reproduction in this region. This study is therefore aimed at establishing the perception, attitude and acceptance of infertile women to multiple pregnancy, single embryo transfer (SET) and multifetal pregnancy reduction (MFPR).

\section{METHODS}

The study was a cross-sectional survey conducted at the IVF unit of the department of Obstetrics and Gynaecology of the University of Benin Teaching hospital and Graceland Medical Centre Benin City. The University of Benin teaching Hospital (UBTH) is one of the major public health institutions in Nigeria with facility and manpower to render IVF services to desiring infertile patients. Graceland Medical Centre (GMC) Benin City is a private health facility with services for assisted reproduction and gynaecologic endoscopy. The study received ethical approval from the hospital ethics and research committee.

The study was conducted over a twelve (12) month period April 2017 to March 2018. All patients recruited for IVF-ET at the UBTH and GMC during the study period who gave informed consent participated. The research instrument was a semi-structured self administered (interviewer guided) questionnaire designed in three sections; Section one is on demography including age and duration of infertility, Section two is on the knowledge of IVF procedure and its complications, Section three is on their perception, attitude and acceptability toward multiple pregnancy and its complications as well as their knowledge, attitude and perception to single embryo transfer and multifetal pregnancy reduction. After explanation of the study objectives as well as the processes involved in SET and MFPR, informed consent was obtained from willing women; they participated in the study in a private setting and were assured that all their information would remain confidential, and their names was not required on the questionnaires.

\section{Statistical analysis}

The appropriately completed questionnaire was analyzed using the SPSS statistical package version 20. Result was aggregated into means and proportions as appropriate and presented as frequency tables and percentages. Subset analysis was performed to determine significant difference between groups and level of significance $(\mathrm{p}<0 / 05)$ was determined with the t-test and chi-square test as appropriate. 


\section{RESULTS}

Seventy-three women participated in the study. According to the below Table 1, the mean age was 39 years, a range of 28 to 53 years. The duration of infertility was between 2 and 22 years with a mean duration of 8.6 years. Majority were nulliparous $50.7 \%$ and $16 \%$ had previously delivered. Similarly majority, $74 \%$ had secondary infertility.

Table 1: Demographic characteristics of participants.

\begin{tabular}{|c|c|c|}
\hline Variable & Frequency (n) & Percent(\%) \\
\hline \multicolumn{2}{|l|}{ Age } & \multirow{3}{*}{ NA } \\
\hline Mean \pm SD & $39.4 \pm 5.8$ & \\
\hline Range & $28-53$ & \\
\hline \multicolumn{2}{|c|}{ Duration of infertility } & \multirow{3}{*}{ NA } \\
\hline Mean \pm SD & $8.7 \pm 4.7$ & \\
\hline Range & $2-22$ & \\
\hline \multicolumn{3}{|l|}{ Parity } \\
\hline 0 & 20 & $27.4 \%$ \\
\hline $0^{+x}$ & 37 & $50 \%$ \\
\hline$\geq 1$ & 16 & $21.9 \%$ \\
\hline \multicolumn{3}{|l|}{ Education } \\
\hline Tertiary & 67 & $91.8 \%$ \\
\hline Secondary & 6 & $8.2 \%$ \\
\hline \multicolumn{3}{|l|}{ Social class } \\
\hline High & 68 & $93.2 \%$ \\
\hline Middle & 5 & $6.8 \%$ \\
\hline \multicolumn{3}{|c|}{ Previous children } \\
\hline Yes & 13 & $17.8 \%$ \\
\hline No & 60 & $82.2 \%$ \\
\hline \multicolumn{3}{|c|}{ Previous IVF } \\
\hline Yes & 32 & $43.8 \%$ \\
\hline No & 41 & $56.2 \%$ \\
\hline \multicolumn{3}{|l|}{ Infertility } \\
\hline Primary & 19 & $26 \%$ \\
\hline Secondary & 54 & $74 \%$ \\
\hline
\end{tabular}

As per the below Table 2 about $70 \%$ of respondents had correct knowledge of IVF and associated risk or complications with IVF. These respondents were familiar with the basic processes of IVF-ET as well as the possible complications like ovarian hyperstimulation syndrome and multiple pregnancies.

\section{Preference and choice of embryo transfer and perception and attitude to SET and MFPR}

Clients choice of number of embryo to be transferred: $1.4 \%$ (one person) for only one embryo transfer; $16.4 \%$ (12) chose two embryos; $58.9 \%$ (43) chose three and $23.3 \%$ (17) chose four embryos.

Majority of respondents (58.9\%) preferred 3 embryos transferred at IVF-ET, they also perceived 3 as the ideal number of embryo to be transferred. Only $3(4.1 \%)$ respondents agreed to have single embryo transferred as the overwhelming majority (70) $95.9 \%$ preferred 2 or more and they felt the more number of embryo transferred the better the chances of achieving pregnancy. Although majority agreed that multiple pregnancy resulting from multiple embryo transfer could result in fetal loss and preterm delivery with eventual lesser live birth rate. Majority (60.3\%) still declined single embryo transfer. According to the Table 3, $44(60.3 \%)$ declined single embryo transfer, 16 (21.9\%) agreed they will probably accept SET with embryo freezing guaranteed as backup. Similarly most respondents, 38.4\% (28) did not accept multifetal pregnancy reduction, but $30.1 \%$ would probably consider it if guaranteed complication free.

Table 2: Participants knowledge of IVF.

\begin{tabular}{|l|l|}
\hline Knowledge variable & Frequency (\%) \\
\hline Knowledge of IVF & \\
\hline Correct & $70(95.9 \%)$ \\
\hline Incorrect & $3(4.1 \%)$ \\
\hline Knowledge of complications of IVF \\
\hline Correct & $65(89 \%)$ \\
\hline Incorrect & $8(11 \%)$ \\
\hline Knows risk of multiple pregnancy \\
\hline Correct & $65(89 \%)$ \\
\hline Incorrect & $8(11 \%)$ \\
\hline
\end{tabular}

Table 3: Participants acceptability of SET and MFPR.

\begin{tabular}{|llllll|}
\hline Variable & Definitely & Probably & Neutral & Probably not & Absolutely not \\
\hline Accept SET & $4(5.5 \%)$ & $10(13.7 \%)$ & $7(9.6 \%)$ & $8(11 \%)$ & $44(60.3 \%)$ \\
\hline Accept MFPR & $4(5.5 \%)$ & $22(30.1 \%)$ & $12(16.4 \%)$ & $7(9.6 \%)$ & $28(38.4 \%)$ \\
\hline $\begin{array}{l}\text { Scared of the } \\
\text { Procedure of MFPR }\end{array}$ & $18(24.2 \%)$ & $21(28.8 \%)$ & $18(24.7 \%)$ & $10(13.7 \%)$ & $6(8.2 \%)$ \\
\hline $\begin{array}{l}\text { Accept MFPR if no } \\
\text { complication }\end{array}$ & $28(38.4 \%)$ & $18(24.7 \%)$ & $11(15.1 \%)$ & $2(2.7 \%)$ & $14(19.2 \%)$ \\
\hline
\end{tabular}




\section{Factors that influence acceptability of SET or MFPR}

According to the Table 4, most respondents considered age $(63 \%)$ and duration of infertility $(78.1 \%)$ as major influencing factor for accepting multiple and rejecting single embryo transfer. Finance $61 \%$, better chance of pregnancy $54.8 \%$ and culture $65.8 \%$ are the other reasons for preferring multiple embryo transfer and rejecting SET. Over $75 \%$ of respondents said they will still accept multiple embryos transferred despite knowledge of the possible complications.

Sub-analysis of age and duration of infertility as a measure of acceptability or not of SET and MFPR showed that non acceptability of SET or MFPR was associated with an older mean age and longer duration of infertility in comparison to those that accepted, but the difference was not statistically significant.

Table 4: Patients perception of factors influencing desire for multiple embryos.

\begin{tabular}{|lll|}
\hline Variable & Frequency $(\mathbf{n}$, & Percent $(\%)$ \\
\hline Age & 47 & 63 \\
\hline Infertility duration & 57 & 78 \\
\hline Finances & 45 & 61.6 \\
\hline To improve outcome & 40 & 54.8 \\
\hline Culture and religion & 48 & 65.8 \\
\hline
\end{tabular}

Table 5: Association of age and infertility duration to the acceptability of SET and MFPR.

\begin{tabular}{|llll|}
\hline Variable SET & Accept SET (no) & Accept SET (yes) & P value \\
\hline Age (mean \pm SD) & $39.9 \pm 5.8$ & $37.9 \pm 5.6$ & 0.22 \\
\hline Duration of infertility (mean \pm SD) & $8.9 \pm 4.7$ & $7.8 \pm 4.6$ & 0.40 \\
\hline MFPR & Accept MFPR (no) & Accept MFPR (yes) & P value \\
\hline Age (mean \pm SD) & $39.7 \pm 5.9$ & $38.9 \pm 5.6$ & 0.57 \\
\hline Duration of infertility (mean \pm SD) & $9.1 \pm 4.8$ & $8.0 \pm 4.5$ & 0.32 \\
\hline
\end{tabular}

SET: Single embryo transfer; MFPR: multifetal pregnancy reduction

\section{DISCUSSION}

It is established that higher complications exist with multiple pregnancy than singleton. ${ }^{7-9}$ Higher order multiple pregnancy resulting from multiple number of embryo transferred, may ultimately result in lower live birth rate. In this study despite an overwhelming majority being knowledgeable of this risk they still preferred to have multiple embryo transferred. Specifically they declined elective single embryo transfer and multifetal pregnancy reduction as strategies to mitigate this complication. While single embryo transfer (SET) and mutifetal pregnancy reduction (MFPR) have been used to reduce incidence of higher order multiple pregnancy and improve the live birth rate following IVF in Europe and America the acceptability of such strategies and protocol is not encouraging in sub-saharan Africa. We found that almost all our respondents declined SET while only about a third agreed to probably accept MFPR. The acceptance of MFPR is premised on the fact that pregnancy has been achieved and thus they may probably consider reducing number of fetuses from 4 or 5 to 2 . In addition the possibility of accepting MFPR was on an assurance of it being a complication free procedure. The ideal choice of number of embryo to be transferred being three (3) for most respondents. This is quite different from the population in Western society where elective SET is routine and widely acceptable. ${ }^{20-21}$ This attitude may be influenced by the Government funding of the ART through the health insurance scheme. ${ }^{22,23}$ Same cannot be said of the situation in Africa where IVF is not covered by insurance and therefore involves huge out-of-pocket cost. This assumption is buttressed in this study where respondents believed that finance may play a role in influencing preference for multiple embryo transferred. So that following painstainking savings for an expensive IVF procedure, the transfer of multiple embryos is hoped will maximize their chance for a successful treatment and answer the question of family size once and for all.

Other factors clients perceived influenced preference for multiple embryo transfer were advanced maternal age, increased duration of infertility, cultural and religious acceptability of multiple births as well as a believe that it will increase their chances of achieving pregnancy. In line with our findings previous research also noted that though clinicians view multiple pregnancy as a problem only few infertile women feel it's a concern. ${ }^{16}$ Similarly the age and duration of infertility have been previously identified as negating factors on acceptance of SET while knowledge of complications of multiple pregnancy and previous experience with preterm delivery positively affected acceptance of MFPR and SET. ${ }^{13,15,16}$ A summation of our review of the acceptability of SET and reduction of multiple fetuses in an infertility population showed that; while most women appear to recognize the associated risks, they are less interested in single embryo transfer because they perceive more embryos transferred as a path to early one time success. The small number of cases and the lack of both multivariate analyses and outcome data limit the power 
and generalization of the clinical implications of our findings.

The desire and hope for a better and improved probability of pregnancy is a principal reason most women elect for transfer of multiple embryos against single embryo transfer. This may suggest that if clients are exposed to other possible factors such as blastocsyt transfer, comprehensive chromosome screening (CCS) or preimplantation genetic screening(PGS) which provides greater opportunity for selection of more viable, better quality and genetically normal embryos and consequently improved pregnancy and live birth rate, then SET may be accepted. The quality of the embryo transferred is important in determining implantation and successful pregnancy. ${ }^{24,25}$ Thus in our setting where resources for chromosomal studies and pre-implantation genetic diagnosis is limited, effort is geared towards achieving a blastocyst stage transfer; This would help select better quality embryo and thus encourage acceptance of counsel for single or maximum of two embryo for transfer.

Government funding or support of IVF services in Nigeria that will result in reduced out-of-pocket cost on the client may improve acceptability of procedures such as SET and MFPR. Furthermore patient education and counseling decision may help in influencing decisions on number of embryo transferred. Previous research documented that educational materials in form of fact sheets, brochures, videos, interactive tools, and patient testimonials reviewing SET and the risks and complications associated with multiple births can help patients make an informed decision on the number of embryos to transfer. ${ }^{26}$

\section{CONCLUSION}

As a consequence of assisted reproduction (IVF), rates multiple births have increased over the years. Despite the risks associated with higher-order multiple births and subsequent calls for single-embryo transfer (SET) or multifetal pregnancy reduction as the standard of care for good-prognosis; most infertility clients and clinicians in sub Saharan Africa still encourage transfer of multiple embryos. Major challenges identified include the strong desire of couples experiencing infertility demanding transfer of multiple embryos in order to maximize their chance for a successful treatment, especially with limited resources. Government funding, client education and a blastocyst transfer protocol may improve acceptability for SET and MFPR as well as preference for less number of embryos transferred in our environment.

Funding: No funding sources

Conflict of interest: None declared

Ethical approval: The study was approved by the Institutional Ethics Committee

\section{REFERENCES}

1. Wang J, Sauer MV. In vitro fertilization (IVF): a review of 3 decades of clinical innovation and technological advancement. Ther Clin Risk Manag. 2006;2:355-364.

2. Audibert C, Glass D. A global perspective on assisted reproductive technology fertility treatment: an 8-country fertility specialist survey. Reprod Biol Endocrinol. 2015;13:133.

3. Mills MS, Eddowes HA, Cahill DJ. A prospective controlled study of in-vitro fertilization, gamete intra-fallopian transfer and intrauterine insemination combined with superovulation. Hum Reprod. 1992;7:490-4.

4. Connolly MP, Hoorens S, Chambers GM. The cost and consequences of assisted reproductive technology: an economic perspective. Hum Reprod Update. 2010;16(6):603-13.

5. Bhatti T, Baibergenova A. A comparison of the costeffectiveness of in vitro fertilization strategies and stimulated intrauterine insemination in a canadian health economic model. Obstet Gynaecol Can. 2008;30(5):411-20.

6. Okohue JE, Onuh SO, Ikimalo JI, Wada I. Patients' preference for number of embryos transferred during IVF/ICSI: A Nigerian experience. Niger J Clin Pract. 2010;13:294-7.

7. Elster N. Less is More: The Risks of Multiple Births. Fertility and Sterility. 2000;74(4):617-23.

8. Mansour RT, Aboughlar MA, serour GI, Amin YM. Multifetal pregnancy reduction: modification of the technique and analysis of the outcome. Fertile Steril. 1999;71:380-4.

9. Blondel B, Kogan MD, Alexander GR. The impact of the increasing number of multiple births on the rates of preterm birth and low birthweight: an international study. Am J Pub Heath. 2002;92(8):1323-30.

10. Hobbins JC. Selective reduction: a perinatal necessity? N Eng J Med. 1988; 318:1062-3.

11. Fujii S, Fukui A, Yamaguchi E. Reducing multiple pregnancies by restricting the number of embryos transferred to two at the first embryo transfer attempt. Hum. Reprod. 1998;13,3550-4.

12. De Sutter P, Van der Elst J, Coetsier T, Dhont M. Single embryo transfer and multiple pregnancy rate reduction in IVF/ICSI: a 5-year appraisal. Reprod Biomed Online. 2003;6:464-9.

13. Henman M, Catt JW, Wood T. Elective transfer of single fresh blastocysts and later transfer of cryostored blastocysts reduces the twin pregnancy rate and can improve the in vitro fertilization live birth rate in younger women. Fertil Steril. 2005;84:1620-7.

14. Tiitinen A, Halttunen M, Härkki P, Vuoristo P, Hydén-Granskog C. Elective single embryo transfer: the value of cryopreservation. Hum Reprod. 2001;16:1140-4. 
15. Ryan GL, Zhang SH, Dokras A. The desire of infertile patients for multiple births. Fertil Steril. 2004;81:500-4.

16. Child T, Henderson AM, Tan SL. The desire for multiple pregnancy in male and female infertility patients. Hum Reprod. 2004;19:558-61.

17. Lynch L, Berkowitz RL, Chitkara U. First trimester transabdominal multifetal pregnancy reduction: a report of 85 cases. Obstet Gynecol. 1990;75:735-8.

18. Yovel L, Yaron Y, Amit A. Embryo reduction in multifetal pregnancies using saline injection: comparison between the transvaginal and transabdominal approach. Hum Reprod. 1992;7:1173-5.

19. Iberico G, Navaro H, Blasco L, Simon C, Pellicer A and Remohi J. Embryo reduction of multifetal pregnancies following assisted reproduction treatment: a modification of the transvaginal ultrasound guided technique. Hum Reprod. 2000;15(10): 2228-33.

20. Kissin DM, Kulkarni AD, Mneimneh A, Warner L, Boulet SL, Crawford S, et al. Embryo transfer practices and multiple births resulting from assisted reproductive technology: an opportunity for prevention. Fertil Steril. 2015;103(4):954-61.

21. Twisk M, van der Veen F, Repping S, Heineman MJ, Korevaar JC, Bossuyt PM. Preferences of subfertile women regarding elective single embryo transfer: additional in vitro fertilization cycles are acceptable, lower pregnancy rates are not. Fertil Steril. 2007;88(4):1006-9.
22. Velez MP, Connolly MP, Kadoch IJ, Phillips S, Bissonnette F. Universal coverage of IVF pays off. Hum Reprod. 2014;29(6):1313-9.

23. Chambers GM, Illingworth PJ, Sullivan EA. Assisted reproductive technology: public funding and the voluntary shift to single embryo transfer in Australia. Med J Aust. 2011;195(10):594-8.

24. Jones GM, Trounson AO, Lolatgis N, Wood C. Factors affecting the success of human blastocsyt development and pregnancy following in vitro fertilization and embryo transfer. Fert Steril. 1998:70(6):1022-9.

25. Swain JE, Carrell D, Cobo A, Meseguer M, Carmen $\mathrm{R}$, Smith GD. Optimizing the culture environment and embryo manipulation to help maintain embryo developmental potential. Fertil Steril. 2016;105(3):571-87.

26. Van Peperstraten A, Nelen W, Grol R, Zielhuis G, Adang E, Stalmeier P, et al. The effect of a multifaceted empowerment strategy on decision making about the number of embryos transferred in in vitro fertilisation: randomised controlled trial. BMJ. 2010;341:c2501.

Cite this article as: Aziken ME, Osaikhuwuomwan JA, Iribhogbe OI. Single embryo transfer and multifetal pregnancy reduction: perception and attitude of women seeking assisted reproduction in Nigeria. Int J Reprod Contracept Obstet Gynecol 2019;8:1821-6. 